\title{
Radiation-induced changes in infra-red spectrum of black zinc diphosphide monocrystals
}

\author{
A.P.Kudin \\ M.P.Dragomanov National Pedagogical University, \\ 9. Pirogova str., Kyiv-30, 252030, Ukraine \\ Tel. 8-044- 221-99-71
}

\begin{abstract}
For the first time the effect of ionizing particles of different type (gamma-quanta, electrons) and neutrons upon infra-red spectra of zinc diphosphide has been studied. It was shown that the defects of phosphorus sublattice, that are the principal centers of optical activity in the infra-red (IR), are responsible for structural evolution of zinc diphosphide crystals in the process interaction with radiation of different types.
\end{abstract}

Keywords: radiation, defects, exciton, annealing, spectral curve, absorption, phosphrous chain.

Paper received 17.09.99; revised manuscript received 14.11.99; accepted for publication 14.03.99.

\section{Introduction}

Zinc diphosphide is an isomorphous semiconductor crystal of $\mathrm{A}^{2} \mathrm{~B}^{5}{ }_{2}$ group with a complex lattice. Black zinc diphosphide being a straight-zone semiconductor with the width of forbidden zone $E_{g}=1.33 \mathrm{eV}$ at $300 \mathrm{~K}$ [1] and having high photosensitivity in near-IR region is a good material for manufacturing quick-response IR light detectors. Studies of IR reflection spectra near the forbidden zone have shown quasi-metal reflection (up to $70 \%$ ) in the maximum of the main band. This corresponds to a free exciton state and to the largest oscillator strength (0.084) from those known at the moment for semiconductors. In recent years free exciton states with $n=2,3,4,5$, $6 \ldots$ and exciton-impurity complexes (EIC) were also found in spectra even at room temperature [2-4]. An oscillator strength of some complexes is $10^{4}$ times larger than that of a free exciton. It was stated that zinc diphosphide is a standard material for studies of various bi-exciton effects. But according to the authors of all abovementioned works, the structure of exciton spectrum (number of lines, their energy location) varied non-controllably from one crystal to another which complicates understanding of their nature. It is known that use of radiation allows to control type and concentration of the introduced defects. An objective of this work is to investigate an effect of radiation-induced disturbances upon IR reflection and absorption spectra of black zinc diphosphide.

\section{Experimental results and discussion}

Crystals of zinc diphosphide (red $\alpha-\mathrm{ZnP}_{2}$, black $\left.\beta-\mathrm{ZnP}_{2}\right)$ were grown from a gaseous phase by the method of static resublimation and had different form depending on certain technological conditions.

Reflection spectra were measured using PGS-2 spectrograph, and IR-reflection spectra - Pye Unicam Fourier spectrometer at $77 \mathrm{~K}$. X-ray structure measurements were made on double-crystal difractometer TURM 62 using goniometers SG-9 and GD-1. The samples were irradiated by $\mathrm{Co}^{60}$ gamma-quanta up to dose $\Phi=5 \times 10^{6} \mathrm{rad}$, electrons with energy $E=1 \mathrm{MeV}$ up to dose $\Phi=1.8 \times 10^{18} \mathrm{~cm}^{-2}$ at $77 \mathrm{~K}$ and thermal neutrons from reactor at room temperature. Low-temperature annealing was carried out using a temperature gradient in special camera based on the Dewar vessel.

Carrier compensations in $\alpha$ - and $\beta$-modifications of crystals are different: for $\alpha-\mathrm{ZnP}_{2}$ a dose dependence is linear in the range $10^{16}-4 \cdot 10^{17} \mathrm{~cm}^{-2}$; for $\beta-\mathrm{ZnP}_{2}$ dose saturation occurs at $\Phi=10^{16} \mathrm{~cm}^{-2}$ (Fig. 1).

The conductivity of $\mathrm{ZnP}_{2}$ irradiated at $300 \mathrm{~K}$ by electrons and neutrons (Fig. 2) is observed to have three stages. The third (higher than $400^{\circ} \mathrm{C}$ ) stage is absent in electronirradiated samples and is connected with regions of disorder induced by neutrons. It can be seen from conductivity recovering curves of $\alpha$ - and $\beta$ - modifications of $\mathrm{ZnP}_{2}$ after isochronous annealing. 


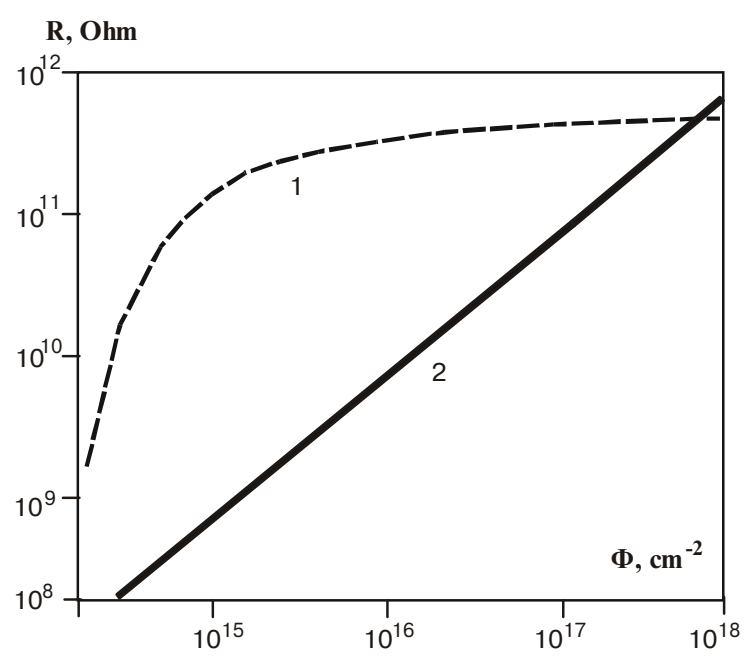

Fig. 1. The resistivity of black (1) and red (2) $\mathrm{ZnP}_{2}$ as the function of electron fluence.

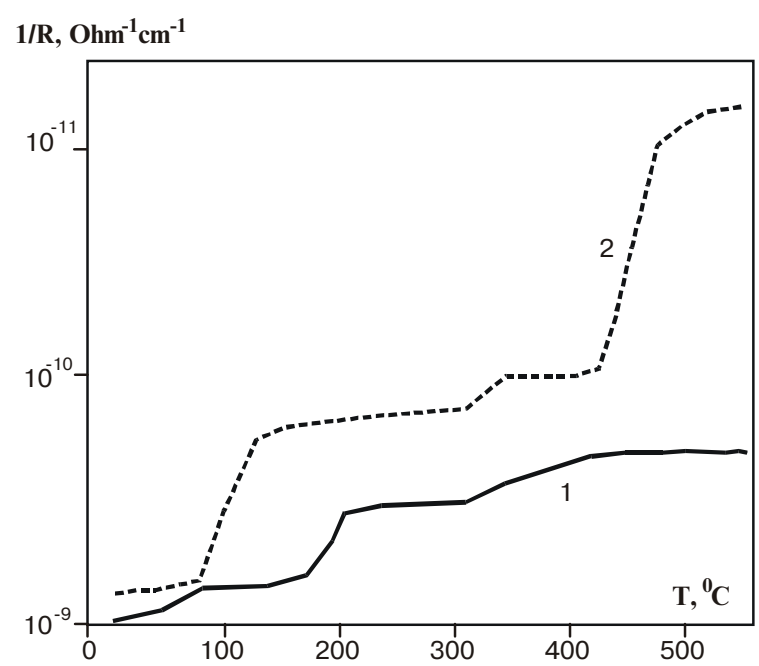

Fig. 2. The conductivity recovering of $\mathrm{ZnP}_{2}$ irradiated at $300 \mathrm{~K}$ by $1 \mathrm{MeV}$ electrons $\left(\Phi=10^{16} \mathrm{~cm}^{-2}\right)$ (curve 1) and by neutrons $\left(\Phi=3 \cdot 10^{16} \mathrm{~cm}^{-2}\right)$ (curve 2).

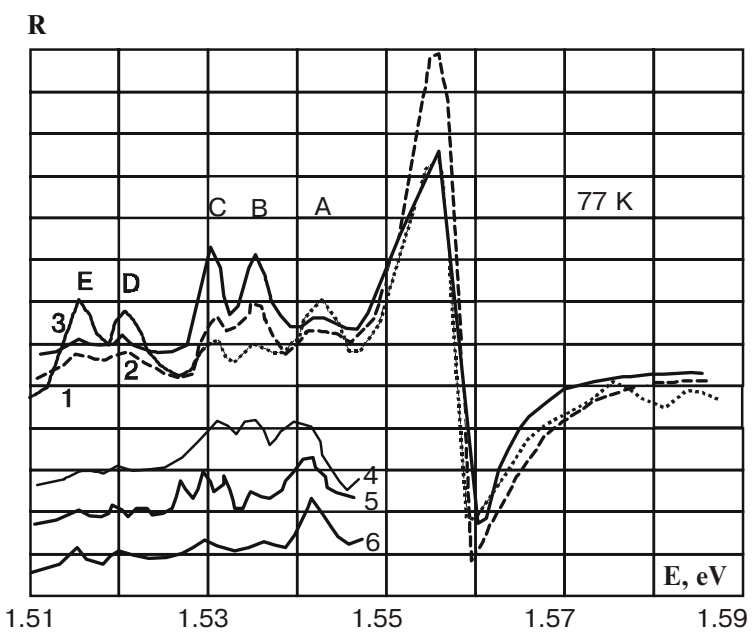

Fig. 3. Black zinc diphosphide reflection spectra: before irradiation (1), irradiated with gamma - quanta (2), and affer annealing at $T=150$ (3), 200 (4), 300 (5), $500 \mathrm{~K}$ (6).
Fig. 3 shows reflection spectra of initial $\mathrm{ZnP}_{2}$ crystals, of those irradiated by gamma-quanta at $77 \mathrm{~K}$ and after low-temperature annealing. Spectrum of initial crystal includes main free exciton band with $n=1(1.560 \mathrm{eV})$ and two other states: $n=2(1.583 \mathrm{eV})$ and $n=3(1.585 \mathrm{eV})$ as well as some bands formed by EIC with energies less than $1.55 \mathrm{eV}$. In the case of irradiation by $\mathrm{Co}^{60}$ gammaquanta at $77 \mathrm{~K}$ an intensity of the main band of free excitons increases by $33 \%$, while an intensity of the band corresponding to the state $n=2$ almost does not change and of $n=3$ decreases down to zero. Small shift of the spectrum (less than $0.001 \mathrm{eV}$ ) towards the infra-red exists. Thus, the result of ionization by gamma-quanta is the formation of separated electron-hole pairs (free excitons) in $\mathrm{ZnP}_{2}$ crystals as it takes plase in the majority of compounds with an ionic chemical bond. The possibility of such mechanism in zinc diphosphide is supported by anomalously low activation energy of the processes of low-temperature defects migration $(0.38-0.74 \mathrm{eV}$ at $T<270 \mathrm{~K}$ [5]), comparatively to the binding energy of exciton as well as by significant part of ionic bond (40\%).

As it is seen in Fig. 3, introducing radiation defects (RD) at $77 \mathrm{~K}$ results in different character of absorption bands in the region of EIC absorption. Really, intensities of $\mathrm{A}, \mathrm{D}$ and $\mathrm{E}$ lines with corresponding energies of 1.5430 , 1.520 and $1.510 \mathrm{eV}$ decrease while intensities of $\mathrm{B}, \mathrm{C}$ lines $(1.530$ and $1.535 \mathrm{eV})$ increase. In the crystals grown with a shift of stoichiometry towards phosphorus, the lines with energies of 1.530, 1.535 and $1.537 \mathrm{eV}$ appeared. Taking into account the studied spectral region, we may suppose that inter-node phosphorus atoms are responsible for an appearance of these lines in initial crystals. As it is seen in Fig. 1, the intensity only of B and C lines with energies which coincide with the above ones increases under irradiation. It means that defects of phosphorus sub-lattice are the optically active centers in IR region of spectrum of $\mathrm{ZnP}_{2}$.

After low-temperature annealing an intensity of free exciton main band restores within the temperature interval $T=100-150 \mathrm{~K}$. Absorption of EIC increases. Thus, the process of free excitons decay accompanied by the formation of bound excitons takes place. It is known that low-energy defect may be formed only as a result of slow interaction of particles. The structure of zinc diphosphide is characterized by high packing density and by several non-equivalent locations of atoms in lattice. That is why free excitons overcoming small distances can localize themselves enough quickly near nodes forming, in such way, autolocalized excitons (quasimolecules) with significant time of localization. As a rule, the localization proceeds through hole exciton component at electrically charged center. The latter may be, for example, the inter-node atom.

To determine the nature of exciton localization center in zinc diphosphide, let us use the results of low-temperature study of individual defects mobility in it [5]. It was found that at temperatures up to $200 \mathrm{~K}$ defects of the type of inter-node phosphorus atoms are annealed. As it is seen in Fig. 3, at $200 \mathrm{~K}$ the intensities of $\mathrm{B}$ and $\mathrm{C}$ bands 


\section{A.P. Kudin: Radiation-induced changes in infra-red spectrum of black zinc ...}

(with energies of 1.530 and $1.535 \mathrm{eV}$ ) restore up to the intensity observed in irradiated sample. So, redistribution of intensities in spectrum at low-temperature annealing within the temperature interval of $100-200 \mathrm{~K}$ is caused by transition of free excitons to the state of bonding with inter-node phosphorus atom. An increase of temperature up to $300 \mathrm{~K}$ results in decrease of intensity of overall spectrum of EIC. Splitting some lines takes place which disappears at $500 \mathrm{~K}$. The similar pattern is found in spectra of crystals irradiated by electrons with an energy $E=1 \mathrm{MeV}$ at room temperature. It is known that decay of electron sub-system can cause not only defects migration and redistribution, but also stimulate the processes of combining the defects and formation of new complexes. As it was shown in earlier, deep acceptor levels correspond to the introduced radiation-induced defects in zinc diphosphide. In accordance with the envelope model of bound EIC proposed by Kirzhenov, the ground states of the complex may be splitted in the case of introducing of deep acceptors. The system of levels is determined by the character of interaction of holes with the acceptor field, its structure, charge state, etc. So, triplet which appears inside the energy interval of $1.525-1.530 \mathrm{eV}$, is caused, a most likely, by the manifesting the center with tetrahedral symmetry. This center may be an acceptor vacancy complex which manifests itself in isochronic annealing of electric conductivity [5] and of narrow component of angular distribution of annihilation quanta in irradiated $\mathrm{ZnP}_{2}$ within the same temperature interval.

Heating of the crystal up to the temperature of $750 \mathrm{~K}$ does not change character of the spectrum while above this temperature we may find sharp shift of free exciton band towards long-wave region of the spectrum (by $0.5 \mathrm{eV})$. This indicates disturbance of crystalline structure which is also confirmed by the X-ray method.

IR-absorption spectra of zinc-diphosphide have shown that small doses of irradiation by electrons influence efficiently a number of phosphorus defects. Investigation of dose effect in broad interval has fixed shift of exciton ground band only by $0.002 \mathrm{eV}$ at $\Phi=1.8 \cdot 10^{18} \mathrm{~cm}^{-2}$.

High-temperature $(T>300 \mathrm{~K})$ isochronic annealing of samples irradiated by electrons $(E=1 \mathrm{MeV})$ at room temperature and by gamma-quanta at $77 \mathrm{~K}$ had the same character. One temperature stage with the central point at $T=500 \mathrm{~K}$ was observed.

It is necessary to mention one special feature of gammairradiated samples after annealing at $500 \mathrm{~K}$. When comparing with the spectra of irradiated crystals, we may see that intensity and width of EIC lines decrease (Fig. 3), and intensity of the main free exciton band increases to some extent. This testifies to some self-ordering of crystalline structure at annealing and restoring of free excitons as an evidence of it. In the samples irradiated by lowenergy electrons and gamma-quanta, the shift of edge of fundamental absorption at $77 \mathrm{~K}$ was not observed. Thus, introducing large doses of point defects exerts low effect on the structure of zinc diphosphide own defects.

Neutron irradiation at room temperature results in increase of absorption coefficient at the edge region and

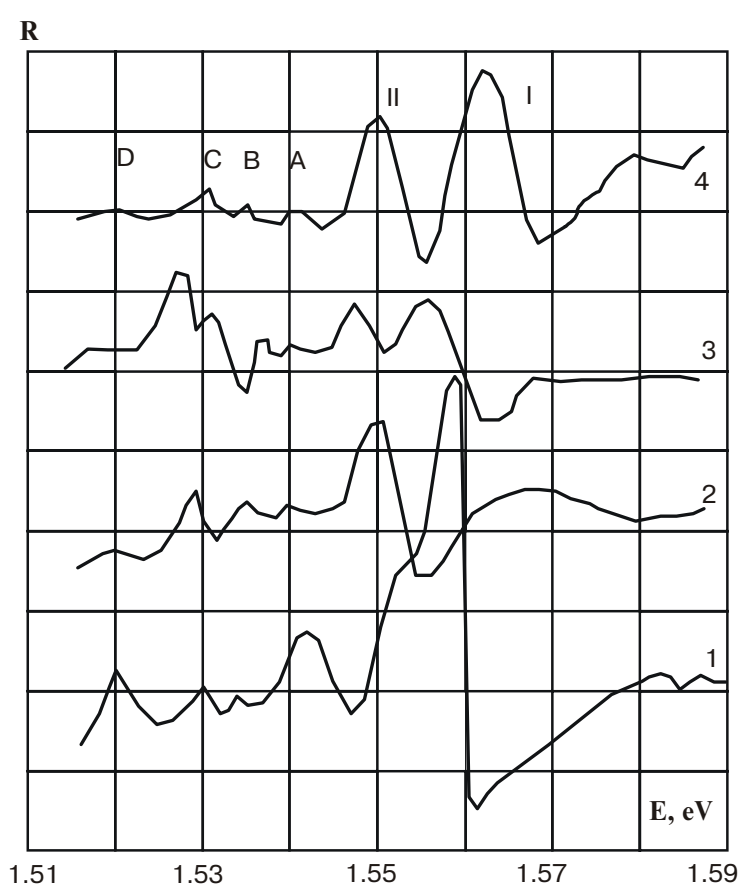

Fig. 4. Black zinc diphosphide reflection spectra: non-irradiated (1), irradiated with neutrons $\Phi=10^{16} \mathrm{~cm}^{-2}(2), \Phi=10^{18} \mathrm{~cm}^{-2}$ (3) and affer annealing at $T=500^{\circ} \mathrm{C}$ (4).

changes significantly exciton spectra (Fig. 4). The special feature of neutron-irradiated zinc diphosphide crystals is the presence of free exciton bands in reflection spectra, as in CdS. This is caused, most likely, by the large Rydberg constant of reversed hydrogen-like series for these crystals $(R=0.702 \mathrm{eV}$ at $77 \mathrm{~K})$. In the case of neutron irradiation, complex defects are mainly introduced, by a type of disordering areas (DA) being the conglomerates of inter-node atoms and vacancies. In contrast to those formed under electron irradiation, they are large-scale disturbances that can form internal electric fields. Exciton scattering on space charges results in decrease of their lifetime. Correspondingly, dispersion at reflection curves decreases, which is observed experimentally.

In comparison with non-irradiated sample, in the case of neutron irradiation by a dose of $\Phi=10^{16} \mathrm{~cm}^{-2}$ (Fig. 4, curve 2), we may find an infra-red shift of the principal spectral lines up to $0.015 \mathrm{eV}$. Change of energy state of free exciton with a dose has linear character. It is significant difference from the temperature effect. This allows us to think that the mechanism of the RD-effect upon free excitons is connected with deformation of crystalline lattice. In the works where an effect of external electric field strength on reflection spectra in structures of metalzinc diphosphide of black modification was studied, it was found that a shift of reflection bands by $0.007 \mathrm{eV}$ was achieved at electric field strength $E=22 \mathrm{kV} / \mathrm{cm}$. It is clear that space DA charge can not cause the shift of bands observed in the experiment. Thus, longwave shift of the spectrum both after neutron irradiation after hightemperature annealing $(T=750 \mathrm{~K})$ of electron-irradi- 
ated samples, is connected, most likely, with structural changes in crystal.

An increase of irradiation dose by two orders more (up to $\Phi=3 \cdot 10^{18} \mathrm{~cm}^{-2}$ ) results in appearance of another spectrum in the area of the free exciton. Two almost equal in intensity lines (I, II) appear, the line II being connected with optical transitions of low oscillator strength (Fig. 4, curve 3).

In [1] where exciton spectra of black zinc diphosphide crystals formed from a melt at different conditions were studied for the first time, two different types of spectra were found. They principally differed only by a number of lines in reflection spectrum connected with transitions from the state of free exciton. In the first case there were two lines with energies of 1.5503 and $1.5610 \mathrm{eV}$ and in the second - one line with the energy of $1.560 \mathrm{eV}$ at $77 \mathrm{~K}$. $\mathrm{X}$-ray structure analysis has shown that the first type of that spectrum is characteristic of monoclinic modification of zinc diphosphide $\mathrm{C}_{2 \mathrm{~h}}$, and the second type - of rhombic modification $\mathrm{D}_{2 \mathrm{~h}}$.

The assumption has been made that an existence of two modifications is a result of formation of doubles during the process of growth from a melt. In spite of doubling structure, studies of optical characteristics of such crystals confirm their high perfection [1]. An analysis of the studies of physical properties of black zinc diphosphide carried out up todate, indicates that the differences of two its modifications were found only in exciton spectra. X-ray structure analysis of such crystals has confirmed the fact of doubling of monoclinic cells ( $a=8.85 \AA, b=7.2 \AA$, $c=7.56 \AA$ ) by raising of the macrostructure symmetry up to rhombic one ( $a=7.48 \AA, b=34.98 \AA, c=7.29 \AA)$. In $\mathrm{X}$-ray goniometric photos, when turning such crystals around the axis $c_{\text {rhomb }}=b_{\text {mon }}$ we may see monoclinic cell with the same $b$ and $c$ parameters, but with twice as much as $a$. Doubling of monoclinic parameter $a$ and increase of reflections in which nodes of two lattices coincide, are caused by overlapping of coordinate grids of monoclinic cells. I.e., the dimensions of doubling area are commensurable with the Bohr radius of exciton . For example, for an exciton with $n=4, R_{c c}=16 \AA$ [3]. That is why an increase of symmetry of lattice becomes visible first of all in exciton spectra.

In accordance with data of chemical analysis of black zinc diphosphide compositions, its rhombic modification, in contrast to the monoclinic one, has larger phosphorus content (by $2 \%$ ) than it is required by stoichiometry. All this allow us to explain redistribution of oscillator strengths between exciton states in the case of neutron irradiation by breaking phosphorus chains, smoothing of crystal stoichiometry and, as a result, by lowering of its symmetry down to the monoclinic one. Can the introduced by an irradiation areas of disordering make this directly? Using the Gossic model for DA we can estimate its average dimension: $R_{D A}=30 \AA$. It was found to be comparable with the dimension of the double for rhombic modification.

So, an irradiation of black zinc diphosphide by thermal neutrons from reactor results in phase transition by the type of displacement in its structure. This conclusion is confirmed by Layegram made in two directions (001) and (100). In non-irradiated crystals we can find three mutually perpendicular planes of symmetry $(\mathrm{mmm})$, and in neutron-irradiated - only one plane $(2 / \mathrm{m})$.

\section{References}

1. V.V. Sobolev, N.N. Syrbu, Optical Spectra and Energy Band Structure of Monoclinic Crystals $\mathrm{ZnP}_{2}$ and $\mathrm{ZnAs}_{2} / /$ Phys. Stat. Sol.(b), 51, pp. 863-872 (1972).

2. M. Sugisaki, M. Nishikawa, O. Arimoto, Emission from the higher members of exciton $(n=2,3,4)$ in $\mathrm{ZnP}_{2} / /$ J.Phys. Soc. Jap., 64 (9), pp. 3506-3513 (1995).

3. M. Sugisaki, O. Arimoto, K. Nakamura, Intersystem conversion between singlet and triplet exciton states in $\mathrm{ZnP}_{2} / / J$. Phys. Soc. Jap., 65 ( 1), pp. $23-26$ (1996).

4. I.S. Gorban, M.M. Biliy, I.M. Dmitruk, Ions of Excitonic Molecule in $\mathrm{ZnP}_{2}$ Crystals // Sol. Stat. Comm., 100 (1), pp. 15 (1996).

5. Z. Januskevicius, V. Koval, A. Kudin, Radiation defects in $\mathrm{ZnP}_{2}$ // Phys. Stat. Sol. 81, pp. 191-194(1984). 